\title{
Analysis of aflatoxin contamination in Myanmar agricultural commodities
}

\author{
Ei Ei Chaw?
}

${ }^{1}$ Commodity Testing and Quality Management Center, Department of Consumer Affairs, Ministry of Commerce, Yangon, Myanmar

\section{Keywords}

aflatoxin; Romer method; thin layer chromatography; visual estimation

\section{Correspondence}

Ei Ei Chaw, Commodity Testing and Quality Management Center, Department of Consumer Affairs, Ministry of Commerce, Yangon, Myanmar.

E-mail: eieichaw1975@gmail.com

(Received April 6, 2017, revised May 31, 2017, accepted June 9, 2017)

\begin{abstract}
This manuscript provides data on the analysis of aflatoxin (AFs) contamination in Myanmar agricultural commodities which were intended for export and domestic consumption from 2008 to 2015 ${ }^{1)}$. Most of the samples were white rice, broken rice, parboiled rice, green mung bean, black sesame seed, white sesame seed, black matpe, butter bean, toor whole, peyin bean (bamboo bean) and yellow maize. The total AFs concentration of these samples was quantitatively analyzed by the Romer method using thin layer chromatography with visual estimation ${ }^{2}$. Aflatoxin (AF) B1 contamination was frequently detected in all of the contaminated samples, however, AFG1 and AFG2 contamination with no AFB1 group was found in one sample of broken rice from 2014. In addition, some samples were contaminated with not only AFB1 but also AFB2 and AFG1. A sample that contained all four kinds of AFs was not found. A 2008 yellow maize sample was found to have the highest concentration of AFB1 $(30.35 \mu \mathrm{g} / \mathrm{kg})$. Generally, the most highly contaminated samples were below the permissible limits for total AF levels as regulated by the European Union and Codex Alimentarius Commission.
\end{abstract}

\section{Introduction}

Myanmar produces different food crops such as cereal grains, oil seeds, pulses and beans. Among them, rice is the staple food and export earning crops of our country. After rice, the other important Myanmar agricultural commodities are oil seeds, pulses and beans. Peanuts, sesame, sunflower and soya bean are the valuable oil seeds in Myanmar. The major types of pulses and beans produced in Myanmar are the green mung bean, butter bean, toor whole, peyin bean (bamboo bean) and black matpe. Yellow maize is one of the cereal grains produced in Myanmar. Most of the Myanmar agricultural commodities are intended for export market but some commodities are used for domestic consumption.

The globalization of food trade has focused on the quality and safety of the imported and exported foods. The climate of Myanmar and geographical location provide favorable conditions of the aflatoxin contamination in agricultural commodities before harvesting (in the field) as well as after harvesting (during storage). Aflatoxin is one of the hazards that may cause food to be unsafe for human consumption. Aflatoxin contamination of food grains especially in agricultural com- modities, is important for food quality and safety. Therefore, in order to food quality control and food safety, there are three government agencies concerned with the food quality control program, namely,
- National Health Lab (NHL)
Food and Drug Administration (FDA)
Department of Health
Ministry of Sports and Health
- Plant Protection Division
Myanmar Agricultural service (MAS)
Ministry of Agriculture, Livestock and Irrigation
- Commodity Testing and Quality Management
Center (CTQM)
Department of Consumer Affairs
Ministry of Commerce.

Myanmar is a developing country without its own standards for laboratory tests and inspection of food grains intended to export markets. Also, the specifications of export agricultural products are based on the buyer's choice. The Ministry of Sports and Health is responsible for the main foodstuffs apart from agricultural products for domestic consumption.

At the CTQM mycotoxin Laboratory, food grain items are tested for aflatoxin contamination to promote export quality. Export promotion is the major 
concern for the economic development of Myanmar since increases in the export of agricultural products makes the price in domestic markets higher. This attracts farmers to grow more. The CTQM mycotoxin laboratory can only detect aflatoxin contamination in agricultural commodities, food and feed. The study and analysis of aflatoxin contamination in Myanmar agricultural commodities can provide data that analyzes contamination by aflatoxin (AF) B1, AFB2, AFG1 and AFG2 in different samples of various commodities from 2008 to 2015 .

\section{Materials and Methods}

The important actors who take part in the supply chain of agricultural commodities in Myanmar are the farmers, primary village collectors, other agents, millers or processors, the town wholesalers, traders and exporters. According to the supply chain, CTQM received test samples from processors, traders and exporters. Sometimes, the inspection agencies such as SGS, OMIC and MITS (Myanmar), sent their samples to the CTQM. The samples used for this study were mixtures of different varieties from various parts of our country.

The samples were analyzed for aflatoxin by the Romer method (1975) using thin layer chromatography (TLC) with visual estimation under UV light. Thin layer chromatography was performed using plates with cilica gel $60 \mathrm{~A}, 20 \mathrm{~cm} \times 20 \mathrm{~cm}$, layer thickness $250 \mu \mathrm{m}$, glass sheets (Whatman) and TLC Silicagel $60 \mathrm{~F}_{254}$ Aluminium sheets, $20 \mathrm{~cm} \times 20 \mathrm{~cm}$ (Merck).

The procedure is performed by making the slurry of the crushed sample and meal with water, extracted by using aqueous acetone and cleaned up with ferric gel, which contains sodium hydroxide, ferric chloride and copper chloride. This method involves comparing the fluorescence intensity of extract spots with those of standard aflatoxin spots. For viewing the TLC spots, a Chromato Vue cabinet equipped with one or more
$15 \mathrm{~W}$, long wave UV lights was adequate. The solvent, acetone-chloroform (1:9) combination was used as the mobile phase (developer). The concentration of aflatoxin $(\mathrm{ppb})$ is calculated by the equation:

\section{$\frac{\text { SxYxV }}{W x Z} \quad$ (ppb)}

where

$S=$ volume in $\mu \mathrm{l}$ of Aflatoxin standard of equivalent intensity to $Z \mu \mathrm{l}$ of sample.

$\mathrm{Y}=$ concentration of Aflatoxin standard in $\mu \mathrm{g} / \mathrm{ml}$

$Z=$ volume in $\mu \mathrm{l}$ of sample extract required, to give fluorescence intensity comparable to that of $S \mu l$ of standard.

$\mathrm{V}=$ volume in $\mu \mathrm{l}$ of solvent required to dilute final extract. $\mathrm{W}=$ weight in $\mathrm{g}$ of original sample contained in final extract.

\section{Results and Discussion}

\section{White Rice}

The aflatoxin contamination levels of white rice analyzed from 2008 to 2015 shows in Table 1. In 2008, AFB1 was detected in 17 out of 24 samples of white rice.

In 2009, 25 samples were positive, out of the 37 samples tested. All of the positive samples contained AFB1, however, two samples contained AFB2 (4.66ppb).

In 2010, 12 out of 22 samples tested positive.

In 2011, there were 62 samples analyzed throughout the year and 49 of those samples were positive. Only 5 samples were contaminated by AFG1 alone.

In 2012, 44 samples out of 84 analyzed were positive.

In 2013, a total of 66 samples were analyzed. Among these, 32 samples were positive.

In 2014, 122 out of the 161 samples tested were positive.

In 2015, 62 total samples were tested, and 43 samples out of the 62 were positive.

Table 1 white rice

\begin{tabular}{|c|c|c|c|c|c|c|c|c|}
\hline Duration & $\begin{array}{c}\text { no of } \\
\text { analyzed } \\
\text { samples }\end{array}$ & $\begin{array}{c}\text { no of } \\
\text { positive } \\
\text { sample }\end{array}$ & $\begin{array}{c}\text { Range } \\
(\mathrm{B} 1) \\
(\mathrm{ppb})\end{array}$ & $\begin{array}{c}\text { Range } \\
(\mathrm{B} 2) \\
(\mathrm{ppb})\end{array}$ & $\begin{array}{c}\text { Range } \\
(\mathrm{G} 1) \\
(\mathrm{ppb})\end{array}$ & $\begin{array}{c}\text { Range } \\
(\mathrm{G} 2) \\
(\mathrm{ppb})\end{array}$ & $\begin{array}{c}\text { Range } \\
\text { (total) } \\
(\mathrm{ppb})\end{array}$ & $\begin{array}{c}\text { Percent of } \\
\text { positive } \\
\text { samples (\%) }\end{array}$ \\
\hline 2008 & 24 & 17 & $4-9$ & - & - & - & $4-9$ & 71 \\
\hline 2009 & 37 & 25 & $0.9-12$ & 5 & - & - & $0.9-14$ & 68 \\
\hline 2010 & 22 & 12 & $0.5-7$ & 1 & - & - & $0.5-7$ & 55 \\
\hline 2011 & 62 & 49 & $0.3-12$ & $0.2-12$ & $0.4-8$ & - & $0.3-12$ & 79 \\
\hline 2012 & 80 & 44 & $0.4-12$ & $0.3-0.9$ & $0.3-0.3$ & - & $0.4-12$ & 55 \\
\hline 2013 & 66 & 32 & $0.6-8$ & 5 & - & - & $0.6-9$ & 48 \\
\hline 2014 & 161 & 122 & $0.8-9$ & $0.9-5$ & - & - & $0.8-9$ & 76 \\
\hline 2015 & 62 & 43 & $2-14$ & $0.9-5$ & 2 & - & $2-19$ & 69 \\
\hline
\end{tabular}

Contamination of Aflatoxin B1, B2, G1, G2 and total AFs range in white rice 


\section{Broken Rice}

Table 2 shows the aflatoxin contamination levels of broken rice analyzed from 2008 to 2015 .

In 2008, AFB1 was detected in 5 out of 10 samples of broken rice.

In 2011, 24 samples tested positive out of 31 samples analyzed.

In 2012, 40 samples were analyzed throughout the year. During the analysis for aflatoxin, 32 samples tested positive.

In 2013, a total of 48 samples were analyzed. Among them, 23 samples were positive.

In 2014, 42 out of 59 samples were positive. One of the positive samples contained only AFG1 and AFG2.

In 2015, the sample total was 54 and 43 samples out of the 54 were positive.

\section{Green Mung Beans}

Table 3 shows the aflatoxin contamination levels of green mung beans analyzed from 2008 to 2015 .

In 2008, none of 11 green mung bean samples detected AFB1.

In 2009, 1 sample tested positive out of 11 samples analyzed.

In 2010, 20 samples out of the 62 tested were positive, with only AFB1 detected.
In 2011, there were 31 green mung bean samples throughout the year and 4 of those samples were positives. Most of the positive samples contained only AFB1.

In 2012, 3 samples were positive out of 18 total samples.

In 2013, 14 samples were analyzed and among them, 2 samples were positive.

In 2014, 16 samples out of 91 samples tested were positive.

In 2015, the total number of samples analyzed was 96 and 38 samples out of the 96 were positive.

\section{Black Sesame seeds}

Table 4 shows the aflatoxin contamination levels of black sesame seeds analyzed from 2008 to 2015 .

In 2008, AFB1 was detected in 33 out of 131 samples of black sesame seeds.

In 2009,70 positive samples were detected out of 204 samples.

In 2010, 37 samples out of 132 were detected as positive.

In 2011, there were 143 black sesame seed samples throughout the year and 26 of those samples were positive.

Table 2 Broken rice

\begin{tabular}{|c|c|c|c|c|c|c|c|c|}
\hline Duration & $\begin{array}{c}\text { no of } \\
\text { analyzed } \\
\text { samples }\end{array}$ & $\begin{array}{c}\text { no of } \\
\text { positive } \\
\text { samples }\end{array}$ & $\begin{array}{c}\text { Range } \\
(\mathrm{B} 1) \\
(\mathrm{ppb})\end{array}$ & $\begin{array}{c}\text { Range } \\
(\mathrm{B} 2) \\
(\mathrm{ppb})\end{array}$ & $\begin{array}{c}\text { Range } \\
(\mathrm{G} 1) \\
(\mathrm{ppb})\end{array}$ & $\begin{array}{c}\text { Range } \\
(\mathrm{G} 2) \\
(\mathrm{ppb})\end{array}$ & $\begin{array}{c}\text { Range } \\
\text { (total) } \\
(\mathrm{ppb})\end{array}$ & $\begin{array}{c}\text { Percent of } \\
\text { positive } \\
\text { samples (\%) }\end{array}$ \\
\hline 2008 & 10 & 5 & $4-4$ & - & - & - & $4-4$ & 50 \\
\hline 2009 & - & - & - & - & - & - & - & - \\
\hline 2010 & - & - & - & - & - & - & - & - \\
\hline 2011 & 31 & 24 & $0.3-12$ & $2-2$ & $4-16$ & - & $0.6-16$ & 77 \\
\hline 2012 & 40 & 32 & $0.3-8$ & $0.4-0.8$ & - & - & $0.4-8$ & 80 \\
\hline 2013 & 48 & 23 & $0.9-12$ & $1-1$ & - & - & $0.9-12$ & 48 \\
\hline 2014 & 59 & 42 & $2-14$ & $0.5-5$ & $14-14$ & $14-14$ & $2-28$ & 71 \\
\hline 2015 & 54 & 43 & $2-14$ & $2-5$ & $4-5$ & - & $2-14$ & 80 \\
\hline
\end{tabular}

Contamination of Aflatoxin $\mathrm{B} 1, \mathrm{~B} 2, \mathrm{G} 1, \mathrm{G} 2$ and total AFs range in broken rice

Table 3 Green Mung Bean

\begin{tabular}{|c|c|c|c|c|c|c|c|c|}
\hline Duration & $\begin{array}{c}\text { no of } \\
\text { analyzed } \\
\text { samples }\end{array}$ & $\begin{array}{c}\text { no of } \\
\text { positive } \\
\text { samples }\end{array}$ & $\begin{array}{c}\text { Range } \\
(\mathrm{B} 1) \\
(\mathrm{ppb})\end{array}$ & $\begin{array}{c}\text { Range } \\
(\mathrm{B} 2) \\
(\mathrm{ppb})\end{array}$ & $\begin{array}{c}\text { Range } \\
(\mathrm{G} 1) \\
(\mathrm{ppb})\end{array}$ & $\begin{array}{c}\text { Range } \\
(\mathrm{G} 2) \\
(\mathrm{ppb})\end{array}$ & $\begin{array}{c}\text { Range } \\
\text { (total) } \\
(\mathrm{ppb})\end{array}$ & $\begin{array}{c}\text { Percent of } \\
\text { positive } \\
\text { samples (\%) }\end{array}$ \\
\hline 2008 & 11 & - & - & - & - & - & - & 0 \\
\hline 2009 & 11 & 1 & $2-2$ & - & - & - & $2-2$ & 9 \\
\hline 2010 & 62 & 20 & $0.3-4$ & - & - & - & $0.3-4$ & 32 \\
\hline 2011 & 31 & 4 & $0.3-3$ & & & - & $0.3-3$ & 13 \\
\hline 2012 & 18 & 3 & $0.4-2$ & - & - & - & $0.4-2$ & 17 \\
\hline 2013 & 14 & 2 & $3-3$ & - & - & - & $3-3$ & 14 \\
\hline 2014 & 91 & 16 & $0.6-4$ & - & - & - & $0.6-3.80$ & 18 \\
\hline 2015 & 96 & 38 & $0.8-8$ & $0.9-6$ & - & - & $2-9$ & 40 \\
\hline
\end{tabular}

Contamination of Aflatoxin B1, B2, G1, G2 and total AFs range in Green Mung Bean 
Table 4 Black Sesame seeds

\begin{tabular}{|c|c|c|c|c|c|c|c|c|}
\hline Duration & $\begin{array}{c}\text { no of } \\
\text { analyzed } \\
\text { samples }\end{array}$ & $\begin{array}{c}\text { no of } \\
\text { positive } \\
\text { samples }\end{array}$ & $\begin{array}{c}\text { Range } \\
(\mathrm{B} 1) \\
(\mathrm{ppb})\end{array}$ & $\begin{array}{c}\text { Range } \\
(\mathrm{B} 2) \\
(\mathrm{ppb})\end{array}$ & $\begin{array}{c}\text { Range } \\
(\mathrm{G} 1) \\
(\mathrm{ppb})\end{array}$ & $\begin{array}{c}\text { Range } \\
(\mathrm{G} 2) \\
(\mathrm{ppb})\end{array}$ & $\begin{array}{c}\text { Range } \\
\text { (total) } \\
(\mathrm{ppb})\end{array}$ & $\begin{array}{c}\text { Percent of } \\
\text { positive } \\
\text { samples (\%) }\end{array}$ \\
\hline 2008 & 131 & 33 & $0.9-5$ & - & - & - & $0.9-5$ & 25 \\
\hline 2009 & 204 & 70 & $0.2-3$ & - & - & - & $0.2-3$ & 34 \\
\hline 2010 & 132 & 37 & $0.3-16$ & - & $0.8-0.8$ & - & $0.3-16$ & 28 \\
\hline 2011 & 143 & 26 & $0.3-7$ & & $0.9-1$ & - & $0.3-7$ & 18 \\
\hline 2012 & 94 & 6 & $0.4-3$ & $0.3-0.3$ & - & - & $0.3-3$ & 6. \\
\hline 2013 & 83 & 6 & $2-2$ & - & - & - & $2-2$ & 7 \\
\hline 2014 & 87 & 18 & $2-9$ & - & $4-4$ & - & $2-9$ & 21 \\
\hline 2015 & 81 & 7 & $2-9$ & $1-1$ & - & - & $2-9$ & 9 \\
\hline
\end{tabular}

Contamination of Aflatoxin B1, B2, G1, G2 and total AFs range in Black Sesame seeds

In 2012, 6 samples were positive out of 94 total samples.

In 2013, 83 samples were analyzed and 6 samples were positive.

In 2014, we tested 18 samples out of 87 samples were positive.

In 2015, the total samples were 81 and 7 samples out of 81 were positive.

\section{White Sesame seeds}

Table 5 shows the aflatoxin contamination levels of white sesame seed analyzed from 2008 to 2015 .

In 2008, 19 samples out of 57 samples of white sesame seeds were detected as positive samples.

In 2009, 6 out of 7 samples tested were positive.

In 2010, 5 samples out of 20 were detected as positive.

In 2011, there were 14 white sesame seed samples throughout the year and 4 samples were positive.

In 2013, 6 samples were analyzed, 1 sample was positive.

In 2014, we tested as no samples out of 2 were positive.

In 2015 , no samples out of 4 samples tested were positive.

\section{Toor Whole}

Table 6 shows the aflatoxin contamination levels of toor whole analyzed from 2008 to 2015.

In 2008, 25 samples out of 120 samples of toor whole were detected as positive samples.

In 2009, none of the 9 samples were positive.

In 2010, 19 samples out of 77 were detected as positive.

In 2011, there were 46 toor whole samples throughout the year. 11 samples were positive.

In 2012, 1 sample was analyzed and that sample was positive.

In 2013, 2014 and 2015, only one sample was tested per year and none of the samples were positive.

\section{Yellow Maize}

Table 7 shows the aflatoxin contamination levels of yellow maize analyzed from 2008 to 2015 .

In 2008,72 samples out of 106 samples of yellow maize were detected as positive samples.

In 2009, we tested 3 numbers of positive samples out of 4 .

In 2011, there were 8 yellow maize samples throughout the year and 7 samples were positive.

In 2012, 11 samples were analyzed and 7 samples were positive.

In 2013, 8 samples out of 8 tested were positive.

\section{Butter bean}

Table 8 shows the aflatoxin contamination level of butter bean analyzed from 2008 to 2015 .

In 2008, AFB1 was detected in 12 out of the 63 butter bean samples.

In 2009, 14 positive samples out of 57 samples were detected.

In 2010, 38 samples out of 72 were identified as positive with AFB1.

In 2011, 82 samples were analyzed throughout the year and 32 samples were positive.

In 2012, 17 samples out of 52 samples were positive.

In 2013, 50 samples were analyzed and among them, 2 samples were positive.

In 2014, 73 samples were tested and 13 samples were positive.

In 2015, 36 samples out of 66 were positive.

\section{Black Matpe}

Table 9 shows the aflatoxin contamination levels of black matpe analyzed from 2008 to 2015 .

In 2008, AFB1 was detected in 7 samples out of the 112 samples of black matpe.

In 2009, 42 samples were tested and 9 were positive.

In 2010, 40 samples out of 164 were detected as positive.

In 2011, 162 samples were analyzed throughout the year and 31 samples were positive. 
Table 5 White Sesame seeds

\begin{tabular}{|c|c|c|c|c|c|c|c|c|}
\hline Duration & $\begin{array}{c}\text { no of } \\
\text { analyzed } \\
\text { samples }\end{array}$ & $\begin{array}{c}\text { no of } \\
\text { positive } \\
\text { samples }\end{array}$ & $\begin{array}{c}\text { Range } \\
(\mathrm{B} 1) \\
(\mathrm{ppb})\end{array}$ & $\begin{array}{c}\text { Range } \\
(\mathrm{B} 2) \\
(\mathrm{ppb})\end{array}$ & $\begin{array}{c}\text { Range } \\
(\mathrm{G} 1) \\
(\mathrm{ppb})\end{array}$ & $\begin{array}{c}\text { Range } \\
(\mathrm{G} 2) \\
(\mathrm{ppb})\end{array}$ & $\begin{array}{c}\text { Range } \\
\text { (total) } \\
(\mathrm{ppb})\end{array}$ & $\begin{array}{c}\text { Percent of } \\
\text { positive } \\
\text { samples (\%) }\end{array}$ \\
\hline 2008 & 57 & 19 & $2-7$ & - & $4-5$ & - & $2-7$ & 33 \\
\hline 2009 & 7 & 6 & $0.3-2$ & - & $2-3$ & - & $0.3-3$ & 86 \\
\hline 2010 & 20 & 5 & $0.3-3$ & - & - & - & $0.3-3$ & 25 \\
\hline 2011 & 14 & 4 & $0.9-4$ & - & - & - & $0.9-4$ & 29 \\
\hline 2012 & - & - & - & - & - & - & - & - \\
\hline 2013 & 6 & 1 & $2-2$ & - & - & - & $2-2$ & 17 \\
\hline 2014 & 2 & - & - & - & - & - & - & 0 \\
\hline 2015 & 4 & - & & & - & - & - & 0 \\
\hline
\end{tabular}

Contamination of Aflatoxin B1, B2, G1, G2 and total AFs range in White Sesame seeds

Table 6 Toor whol

\begin{tabular}{|c|c|c|c|c|c|c|c|c|}
\hline Duration & $\begin{array}{c}\text { no of } \\
\text { analyzed } \\
\text { samples }\end{array}$ & $\begin{array}{c}\text { no of } \\
\text { positive } \\
\text { samples }\end{array}$ & $\begin{array}{c}\text { Range } \\
(\mathrm{B} 1) \\
(\mathrm{ppb})\end{array}$ & $\begin{array}{c}\text { Range } \\
(\mathrm{B} 2) \\
(\mathrm{ppb})\end{array}$ & $\begin{array}{c}\text { Range } \\
(\mathrm{G} 1) \\
(\mathrm{ppb})\end{array}$ & $\begin{array}{c}\text { Range } \\
(\mathrm{G} 2) \\
(\mathrm{ppb})\end{array}$ & $\begin{array}{c}\text { Range } \\
\text { (total) } \\
(\mathrm{ppb})\end{array}$ & $\begin{array}{c}\text { Percent of } \\
\text { positive } \\
\text { samples (\%) }\end{array}$ \\
\hline 2008 & 120 & 25 & $2-11$ & - & - & - & $2-11$ & 21 \\
\hline 2009 & 9 & - & - & - & - & - & - & 0 \\
\hline 2010 & 77 & 19 & $0.3-5$ & - & - & - & $0.3-5$ & 25 \\
\hline 2011 & 46 & 11 & $0.3-4$ & - & - & - & $0.3-4$ & 24 \\
\hline 2012 & 1 & 1 & $4-4$ & - & - & - & $4-4$ & 100 \\
\hline 2013 & 1 & - & - & - & - & - & - & 0 \\
\hline 2014 & 1 & - & - & - & - & - & - & 0 \\
\hline 2015 & 1 & - & & & - & - & - & 0 \\
\hline
\end{tabular}

Contamination of Aflatoxin B1, B2, G1, G2 and total AFs range in Toor whole

Table 7 Yellow Maize

\begin{tabular}{|c|c|c|c|c|c|c|c|c|}
\hline Duration & $\begin{array}{c}\text { no of } \\
\text { analyzed } \\
\text { samples }\end{array}$ & $\begin{array}{c}\text { no of } \\
\text { positive } \\
\text { samples }\end{array}$ & $\begin{array}{c}\text { Range } \\
(\mathrm{B} 1) \\
(\mathrm{ppb})\end{array}$ & $\begin{array}{c}\text { Range } \\
(\mathrm{B} 2) \\
(\mathrm{ppb})\end{array}$ & $\begin{array}{c}\text { Range } \\
(\mathrm{G} 1) \\
(\mathrm{ppb})\end{array}$ & $\begin{array}{c}\text { Range } \\
(\mathrm{G} 2) \\
(\mathrm{ppb})\end{array}$ & $\begin{array}{c}\text { Range } \\
\text { (total) } \\
(\mathrm{ppb})\end{array}$ & $\begin{array}{c}\text { Percent of } \\
\text { positive } \\
\text { samples (\%) }\end{array}$ \\
\hline 2008 & 106 & 72 & $2-30$ & $2-4$ & $3-9$ & - & $2-30$ & 68 \\
\hline 2009 & 4 & 3 & $8-19$ & - & - & - & $8-19$ & 75 \\
\hline 2010 & - & - & - & - & - & - & - & - \\
\hline 2011 & 8 & 7 & $0.5-9$ & - & $2-3$ & $0.3-0.3$ & $0.5-9$ & 88 \\
\hline 2012 & 11 & 7 & $3-8$ & - & $9-9$ & - & $3-9$ & 64 \\
\hline 2013 & 8 & 8 & $2-6$ & - & - & - & $2-6$ & 100 \\
\hline 2014 & - & - & - & - & - & - & - & - \\
\hline 2015 & - & - & & & - & - & - & - \\
\hline
\end{tabular}

Contamination of Aflatoxin B1, B2, G1, G2 and total AFs range in Yellow Maize 
Table 8 Butter bean

\begin{tabular}{|c|c|c|c|c|c|c|c|c|}
\hline Duration & $\begin{array}{c}\text { no of } \\
\text { analyzed } \\
\text { samples }\end{array}$ & $\begin{array}{c}\text { no of } \\
\text { positive } \\
\text { samples }\end{array}$ & $\begin{array}{c}\text { Range } \\
\text { (B1) } \\
\text { (ppb) }\end{array}$ & $\begin{array}{c}\text { Range } \\
\text { (B2) } \\
\text { (ppb) }\end{array}$ & $\begin{array}{c}\text { Range } \\
\text { (G1) } \\
\text { (ppb) }\end{array}$ & $\begin{array}{c}\text { Range } \\
\text { (G2) } \\
\text { (ppb) }\end{array}$ & $\begin{array}{c}\text { Range } \\
\text { (total) } \\
\text { (ppb) }\end{array}$ & $\begin{array}{c}\text { Percent of } \\
\text { positive } \\
\text { samples (\%) }\end{array}$ \\
\hline 2008 & 63 & 12 & $2-7$ & - & - & - & $2-7$ & 19 \\
\hline 2009 & 57 & 14 & $2-6$ & $2-2$ & - & - & $2-8$ & 25 \\
\hline 2010 & 72 & 38 & $0.3-6$ & - & - & - & $0.3-6$ & 53 \\
\hline 2011 & 82 & 32 & $0.3-8$ & - & - & - & $0.3-8$ & 39 \\
\hline 2012 & 52 & 17 & $0.4-8$ & - & - & - & $0.4-8$ & 33 \\
\hline 2013 & 50 & 2 & $3-6$ & - & - & - & $3-6$ & 4 \\
\hline 2014 & 73 & 13 & $0.8-8$ & - & - & - & $0.8-8$ & 18 \\
\hline 2015 & 66 & 36 & $2-14$ & $0.9-1$ & - & - & $2-15$ & 55 \\
\hline
\end{tabular}

Contamination of Aflatoxin B1, B2, G1, G2 and total AFs range in Butter bean

Table 9 Black Matpe

\begin{tabular}{|c|c|c|c|c|c|c|c|c|}
\hline Duration & $\begin{array}{c}\text { no of } \\
\text { analyzed } \\
\text { samples }\end{array}$ & $\begin{array}{c}\text { no of } \\
\text { positive } \\
\text { samples }\end{array}$ & $\begin{array}{c}\text { Range } \\
\text { (B1) } \\
\text { (ppb) }\end{array}$ & $\begin{array}{c}\text { Range } \\
\text { (B2) } \\
\text { (ppb) }\end{array}$ & $\begin{array}{c}\text { Range } \\
\text { (G1) } \\
\text { (ppb) }\end{array}$ & $\begin{array}{c}\text { Range } \\
(G 2) \\
(p p b)\end{array}$ & $\begin{array}{c}\text { Range } \\
\text { (total) } \\
\text { (ppb) }\end{array}$ & $\begin{array}{c}\text { Percent of } \\
\text { positive } \\
\text { samples (\%) }\end{array}$ \\
\hline 2008 & 112 & 7 & 4-11 & - & - & - & $4-11$ & 6 \\
\hline 2009 & 42 & 9 & $0.3-8$ & $2-2$ & - & - & $0.3-10$ & 21 \\
\hline 2010 & 164 & 40 & $0.3-4$ & - & - & - & $0.3-4$ & 24 \\
\hline 2011 & 162 & 31 & $0.3-8$ & - & - & - & $0.3-8$ & 19 \\
\hline 2012 & 68 & 7 & $0.9-4$ & - & - & - & $0.9-4$ & 10 \\
\hline 2013 & 74 & 2 & 4- 4 & - & - & - & $4-4$ & 3 \\
\hline 2014 & 21 & 6 & $2-9$ & - & - & - & $2-9$ & 29 \\
\hline 2015 & 23 & 2 & $2-11$ & - & - & - & $2-11$ & 9 \\
\hline
\end{tabular}

Contamination of Aflatoxin B1, B2, G1, G2 and total AFs range in Black Matpe

In 2012, 7 samples were positive out of 68 total samples.

In 2013, 74 samples were analyzed and 2 samples were positive.

In 2014, 6 out of 21 samples tested were positive.

In 2015, 2 samples out of 23 were positive.

\section{Peyin (Bamboo Bean)}

Table 10 shows the aflatoxin contamination levels of peyin beans or bamboo beans analyzed from 2008 to 2015 .

In 2008 , none of the samples out of 5 were detected as positive samples.

In 2009, 1 sample was identified as AFB1 contaminated.

In 2010, 4 samples out of 8 were detected as positive.

In 2011, 15 samples were analyzed throughout the year and 6 samples were positive.

In 2012, 10 samples were analyzed with 7 of the samples positive.

In 2013, 2 samples were positive out of 6 samples tested.

In 2014, 6 samples were tested and 2 samples were positive.

In 2015 , the total number of samples tested was 3 and 1 sample was positive.

\section{Red Kidney Beans}

Table 11 shows the aflatoxin contamination levels of red kidney beans analyzed from 2008 to 2015 .

In 2008, none of the 4 samples were positive for AFB1.

In 2014, 14 samples were tested 9 were positive.

In 2015 , 2. total samples were analyzed and no positive sample were detected.

\section{Sultanipya}

Table 12 shows the aflatoxin contamination levels of Sultanipya analyzed from 2008 to 2015.

In 2008, AFB1 was detected in 3 samples out of the 6 Sultanipya samples.

In 2011, none of the 3 samples were positive.

In 2012, 2 samples were positive out of 5 .

In 2013, 4 samples were analyzed and none were positive.

In 2014, 6 samples were analyzed and 2 samples were positive.

In 2015, 3 samples out of 5 were positive.

\section{Black eyed beans}

Table 13 shows the aflatoxin contaminated levels of black eyed beans analyzed from 2008 to 2015 . 
Table 10 Peyin (Bamboo Bean)

\begin{tabular}{|c|c|c|c|c|c|c|c|c|}
\hline Duration & $\begin{array}{c}\text { no of } \\
\text { analyzed } \\
\text { samples }\end{array}$ & $\begin{array}{c}\text { no of } \\
\text { positive } \\
\text { samples }\end{array}$ & $\begin{array}{c}\text { Range } \\
(\mathrm{B} 1) \\
(\mathrm{ppb})\end{array}$ & $\begin{array}{c}\text { Range } \\
(\mathrm{B} 2) \\
(\mathrm{ppb})\end{array}$ & $\begin{array}{c}\text { Range } \\
(\mathrm{G} 1) \\
(\mathrm{ppb})\end{array}$ & $\begin{array}{c}\text { Range } \\
(\mathrm{G} 2) \\
(\mathrm{ppb})\end{array}$ & $\begin{array}{c}\text { Range } \\
\text { (total) } \\
(\mathrm{ppb})\end{array}$ & $\begin{array}{c}\text { Percent of } \\
\text { positive } \\
\text { samples (\%) }\end{array}$ \\
\hline 2008 & 5 & - & - & - & - & - & - & 0 \\
\hline 2009 & 5 & 1 & $2-2$ & - & - & - & $2-2$ & 20 \\
\hline 2010 & 8 & 4 & $0.6-2$ & - & - & - & $0.6-2$ & 50 \\
\hline 2011 & 15 & 6 & $0.3-8$ & - & - & - & $0.3-8$ & 40 \\
\hline 2012 & 10 & 7 & $0.6-8$ & - & - & - & $0.6-8$ & 70 \\
\hline 2013 & 6 & 2 & $2-2$ & - & - & - & $2-2$ & 33 \\
\hline 2014 & 6 & 2 & $0.8-2$ & - & - & - & $0.8-2$ & 33 \\
\hline 2015 & 3 & 1 & $4-4$ & - & - & - & $4-4$ & 33 \\
\hline
\end{tabular}

Contamination of Aflatoxin B1, B2, G1,G2 and total AFs range in Peyin Bean

Table 11 Red Kidney Bean

\begin{tabular}{|c|c|c|c|c|c|c|c|c|}
\hline Duration & $\begin{array}{c}\text { no of } \\
\text { analyzed } \\
\text { samples }\end{array}$ & $\begin{array}{c}\text { no of } \\
\text { positive } \\
\text { samples }\end{array}$ & $\begin{array}{c}\text { Range } \\
(\mathrm{B} 1) \\
(\mathrm{ppb})\end{array}$ & $\begin{array}{c}\text { Range } \\
(\mathrm{B} 2) \\
(\mathrm{ppb})\end{array}$ & $\begin{array}{c}\text { Range } \\
(\mathrm{G} 1) \\
(\mathrm{ppb})\end{array}$ & $\begin{array}{c}\text { Range } \\
(\mathrm{G} 2) \\
(\mathrm{ppb})\end{array}$ & $\begin{array}{c}\text { Range } \\
\text { (total) } \\
(\mathrm{ppb})\end{array}$ & $\begin{array}{c}\text { Percent of } \\
\text { positive } \\
\text { samples (\%) }\end{array}$ \\
\hline 2008 & 4 & - & - & - & - & - & - & 0 \\
\hline 2009 & - & - & - & - & - & - & - & - \\
\hline 2010 & - & - & - & - & - & - & - & - \\
\hline 2011 & - & - & - & - & - & - & - & - \\
\hline 2012 & - & - & - & - & - & - & - & - \\
\hline 2013 & - & - & - & - & - & - & - & - \\
\hline 2014 & 14 & 9 & $2-11$. & $1-1$ & - & - & $2-12$ & 64 \\
\hline 2015 & 2 & - & - & - & - & - & - & 0 \\
\hline
\end{tabular}

Contamination of Aflatoxin B1, B2, G1, G2 and total AFs range in Red Kidney Bean

Table 12 Sul tanipya

\begin{tabular}{|c|c|c|c|c|c|c|c|c|}
\hline Duration & $\begin{array}{c}\text { no of } \\
\text { analyzed } \\
\text { samples }\end{array}$ & $\begin{array}{c}\text { no of } \\
\text { positive } \\
\text { samples }\end{array}$ & $\begin{array}{c}\text { Range } \\
(\mathrm{B} 1) \\
(\mathrm{ppb})\end{array}$ & $\begin{array}{c}\text { Range } \\
(\mathrm{B} 2) \\
(\mathrm{ppb})\end{array}$ & $\begin{array}{c}\text { Range } \\
(\mathrm{G} 1) \\
(\mathrm{ppb})\end{array}$ & $\begin{array}{c}\text { Range } \\
(\mathrm{G} 2) \\
(\mathrm{ppb})\end{array}$ & $\begin{array}{c}\text { Range } \\
\text { (total) } \\
(\mathrm{ppb})\end{array}$ & $\begin{array}{c}\text { Percent of } \\
\text { positive } \\
\text { samples (\%) }\end{array}$ \\
\hline 2008 & 6 & 3 & $3-7$ & - & - & - & $3-7$ & 50 \\
\hline 2009 & - & - & - & - & - & - & - & - \\
\hline 2010 & - & - & - & - & - & - & - & - \\
\hline 2011 & 3 & - & - & - & - & - & - & 0 \\
\hline 2012 & 5 & 2 & $3-8$ & - & - & - & $3-8$ & 40 \\
\hline 2013 & 4 & - & - & - & - & - & - & 0 \\
\hline 2014 & 6 & 2 & $2-4$ & - & - & - & $2-4$ & 33 \\
\hline 2015 & 5 & 3 & $4-6$ & - & - & - & $4-6$ & 60 \\
\hline
\end{tabular}

Contamination of Aflatoxin B1, B2, G1, G2 and total AFs range in Sul tanipya 
Table 13 Black eyed bean

\begin{tabular}{|c|c|c|c|c|c|c|c|c|}
\hline Duration & $\begin{array}{c}\text { no of } \\
\text { analyzed } \\
\text { samples }\end{array}$ & $\begin{array}{c}\text { no of } \\
\text { positive } \\
\text { samples }\end{array}$ & $\begin{array}{c}\text { Range } \\
(\mathrm{B} 1) \\
(\mathrm{ppb})\end{array}$ & $\begin{array}{c}\text { Range } \\
(\mathrm{B} 2) \\
(\mathrm{ppb})\end{array}$ & $\begin{array}{c}\text { Range } \\
(\mathrm{G} 1) \\
(\mathrm{ppb})\end{array}$ & $\begin{array}{c}\text { Range } \\
(\mathrm{G} 2) \\
(\mathrm{ppb})\end{array}$ & $\begin{array}{c}\text { Range } \\
\text { (total) } \\
(\mathrm{ppb})\end{array}$ & $\begin{array}{c}\text { Percent of } \\
\text { positive } \\
\text { samples (\%) }\end{array}$ \\
\hline 2008 & 2 & - & - & - & - & - & - & 0 \\
\hline 2009 & - & - & - & - & - & - & - & - \\
\hline 2010 & - & - & - & - & - & - & - & - \\
\hline 2011 & - & - & - & - & - & - & - & - \\
\hline 2012 & - & - & - & - & - & - & - & - \\
\hline 2013 & - & - & - & - & - & - & - & - \\
\hline 2014 & 6 & - & - & - & - & - & - & 0 \\
\hline 2015 & 12 & 5 & $2-9$ & - & - & - & $2-9$ & 42 \\
\hline
\end{tabular}

Contamination of Aflatoxin B1, B2, G1, G2 and total AFs range in Black eyed_bean

In 2008 , we detected no aflatoxin contaminated samples of black eyed beans out of 2 samples.

In 2014, we tested 6 samples but no were positive.

In 2015 , the total number of samples analyzed was 12 and 5 samples were positive.

\section{Bocate beans (Cow Peas)}

Table 14 shows the aflatoxin contamination levels of bocate beans (cow peas) analyzed from 2008 to 2015.

In 2008, we did not detect any aflatoxin contaminated sample out of the 5 samples of bocate beans tested.

In 2011 and 2012, there was only one sample for each year and aflatoxin contaminated sample was not detected.

In 2013, 3 samples were analyzed and among them, 2 samples were positive.

In 2014, we tested as 5 samples out of 12 samples were positive.

In 2015, the total samples were 3 and one sample out of 3 was positive.

\section{Soy beans}

Table 15 shows the aflatoxin contamination levels of soy beans analyzed from 2008 to 2015 .

In 2008, no aflatoxin was detected in one samples of soy bean analyzed.

In 2012, we detected no aflatoxin contamination in only sample of soy bean.

In 2014, we detected no aflatoxin in three samples of soy bean that were analyzed.

In 2015, 3 samples out of 4 samples were positive.

\section{Chicken pea beans}

Table 16 shows the aflatoxin contamination levels of chicken pea beans analyzed from 2008 to 2015 .

In 2008, one sample out of 2 analyzed was positive.

In 2014, 1 sample was tested and no aflatoxin contamination was found.

In 2015, 3 samples were positive.

\section{Peanut cake}

Table 17 shows the aflatoxin contamination levels of Peanut cake analyzed from 2008 to 2015.

In 2008, 2 samples out of 6 detected AFB1.

In 2011, 2 samples were analyzed throughout the year and both samples were positive.

In 2012, one sample was aflatoxin contaminated.

In 2013, 2 samples out of 5 samples were positive.

\section{Parboiled rice}

Table 18 shows the aflatoxin contamination levels of parboiled rice analyzed from 2008 to 2015 .

In 2012, 8 samples were positive out of 15 .

In 2013, 10 samples were analyzed and 2 were positive.

In 2014, 53 samples were tested and 38 samples were positive.

In 2015, 36 samples out of 53 were positive.

\section{Discussion}

A summary the aflatoxin contamination of agricultural commodities in Myanmar from 2008 to 2015 are shown in Table 19. From Table 19, it can be seen that 1409 samples were detected as aflatoxin contaminated out of the 4042 samples analyzed eight-year study period. Although most of 4042 samples were intended for export markets, some of them were used for domestic consumption (as raw) for snack and beverages factories and animal feeds.

Maize was the most aflatoxin contaminated commodity followed by broken rice and white rice as the second most aflatoxin contaminated commodities, while parboiled rice was the third most aflatoxin contaminated commodity in Myanmar during eight-year (2008-2015) study period. All of the positive samples contained mostly AFB1 and the other aflatoxin were extent.

Analysis of aflatoxin contamination in Myanmar agricultural commodities from the eight-year study showed that most of the contaminated samples were below the regulated limits of European Union (EU) and Codex Alimentarius Commission for AFB1 contamination. 
Table 14 Bocate bean (Cow Pea)

\begin{tabular}{|c|c|c|c|c|c|c|c|c|}
\hline Duration & $\begin{array}{c}\text { no of } \\
\text { analyzed } \\
\text { samples }\end{array}$ & $\begin{array}{c}\text { no of } \\
\text { positive } \\
\text { samples }\end{array}$ & $\begin{array}{c}\text { Range } \\
(\mathrm{B} 1) \\
(\mathrm{ppb})\end{array}$ & $\begin{array}{c}\text { Range } \\
(\mathrm{B} 2) \\
(\mathrm{ppb})\end{array}$ & $\begin{array}{c}\text { Range } \\
(\mathrm{G} 1) \\
(\mathrm{ppb})\end{array}$ & $\begin{array}{c}\text { Range } \\
(\mathrm{G} 2) \\
(\mathrm{ppb})\end{array}$ & $\begin{array}{c}\text { Range } \\
\text { (total) } \\
(\mathrm{ppb})\end{array}$ & $\begin{array}{c}\text { Percent of } \\
\text { positive } \\
\text { samples (\%) }\end{array}$ \\
\hline 2008 & 5 & - & - & - & - & - & - & 0 \\
\hline 2009 & - & - & - & - & - & - & - & - \\
\hline 2010 & - & - & - & - & - & - & - & - \\
\hline 2011 & 1 & - & - & - & - & - & - & 0 \\
\hline 2012 & 1 & - & - & - & - & - & - & 0 \\
\hline 2013 & 3 & 2 & $2-2$ & - & - & - & $2-2$ & 67 \\
\hline 2014 & 12 & 5 & $0.9-4$ & - & - & - & $0.9-4$ & 42 \\
\hline 2015 & 3 & 1 & $2-2$ & - & - & - & $2-2$ & 33 \\
\hline
\end{tabular}

Contamination of Aflatoxin B1, B2, G1, G2 and total AFs range in Bocate bean

Table 15 Soy bean

\begin{tabular}{|c|c|c|c|c|c|c|c|c|}
\hline Duration & $\begin{array}{c}\text { no of } \\
\text { analyzed } \\
\text { samples }\end{array}$ & $\begin{array}{c}\text { no of } \\
\text { positive } \\
\text { samples }\end{array}$ & $\begin{array}{c}\text { Range } \\
(\mathrm{B} 1) \\
(\mathrm{ppb})\end{array}$ & $\begin{array}{c}\text { Range } \\
(\mathrm{B} 2) \\
(\mathrm{ppb})\end{array}$ & $\begin{array}{c}\text { Range } \\
(\mathrm{G} 1) \\
(\mathrm{ppb})\end{array}$ & $\begin{array}{c}\text { Range } \\
(\mathrm{G} 2) \\
(\mathrm{ppb})\end{array}$ & $\begin{array}{c}\text { Range } \\
\text { (total) } \\
(\mathrm{ppb})\end{array}$ & $\begin{array}{c}\text { Percent of } \\
\text { positive } \\
\text { samples (\%) }\end{array}$ \\
\hline 2008 & 1 & - & - & - & - & - & - & 0 \\
\hline 2009 & - & - & - & - & - & - & - & - \\
\hline 2010 & - & - & - & - & - & - & - & - \\
\hline 2011 & - & - & - & - & - & - & - & - \\
\hline 2012 & 1 & - & - & - & - & - & - & 0 \\
\hline 2013 & - & - & - & - & - & - & - & - \\
\hline 2014 & 3 & - & - & - & - & - & - & 0 \\
\hline 2015 & 4 & 3 & $2-5$ & - & - & - & $2-5$ & 75 \\
\hline
\end{tabular}

Contamination of Aflatoxin B1, B2, G1, G2 and total AFs range in Soy bean

Table 16 Chicken pea bean

\begin{tabular}{|c|c|c|c|c|c|c|c|c|}
\hline Duration & $\begin{array}{c}\text { no of } \\
\text { analyzed } \\
\text { samples }\end{array}$ & $\begin{array}{c}\text { no of } \\
\text { positive } \\
\text { samples }\end{array}$ & $\begin{array}{c}\text { Range } \\
(\mathrm{B} 1)\end{array}$ & $\begin{array}{c}\text { Range } \\
(\mathrm{B} 2)\end{array}$ & $\begin{array}{c}\text { Range } \\
(\mathrm{G} 1)\end{array}$ & $\begin{array}{c}\text { Range } \\
(\mathrm{G} 2)\end{array}$ & $\begin{array}{c}\text { Range } \\
\text { (total) }\end{array}$ & $\begin{array}{c}\text { Percent of } \\
\text { positive } \\
\text { samples (\%) }\end{array}$ \\
\hline 2008 & 2 & 1 & $4-4$ & - & - & - & $4-4$ & 50 \\
\hline 2009 & - & - & - & - & - & - & - & - \\
\hline 2010 & - & - & - & - & - & - & - & - \\
\hline 2011 & - & - & - & - & - & - & - & - \\
\hline 2012 & - & - & - & - & - & - & - & - \\
\hline 2013 & - & - & - & - & - & - & - & - \\
\hline 2014 & 1 & - & - & - & - & - & - & 0 \\
\hline 2015 & 4 & 3 & $4-7$ & - & - & - & $4-7$ & 75 \\
\hline
\end{tabular}

Contamination of Aflatoxin B1, B2, G1, G2 and total AFs range in Chicken pea bean 
Table 17 Peanut cake

\begin{tabular}{|c|c|c|c|c|c|c|c|c|}
\hline Duration & $\begin{array}{c}\text { no of } \\
\text { analyzed } \\
\text { samples }\end{array}$ & $\begin{array}{c}\text { no of } \\
\text { positive } \\
\text { samples }\end{array}$ & $\begin{array}{c}\text { Range } \\
(\mathrm{B} 1) \\
(\mathrm{ppb})\end{array}$ & $\begin{array}{c}\text { Range } \\
(\mathrm{B} 2) \\
(\mathrm{ppb})\end{array}$ & $\begin{array}{c}\text { Range } \\
(\mathrm{G} 1) \\
(\mathrm{ppb})\end{array}$ & $\begin{array}{c}\text { Range } \\
(\mathrm{G} 2) \\
(\mathrm{ppb})\end{array}$ & $\begin{array}{c}\text { Range } \\
\text { (total) } \\
(\mathrm{ppb})\end{array}$ & $\begin{array}{c}\text { Percent of } \\
\text { positive } \\
\text { samples (\%) }\end{array}$ \\
\hline 2008 & 6 & 2 & $46-50$ & - & - & - & $46-50$ & 33 \\
\hline 2009 & - & - & - & - & - & - & - & - \\
\hline 2010 & - & - & - & - & - & - & - & - \\
\hline 2011 & 2 & 2 & $8-24$ & $2-2$ & $8-8$ & - & $10-32$ & 100 \\
\hline 2012 & 1 & 1 & - & $2-2$ & - & - & $2-2$ & 100 \\
\hline 2013 & 5 & 2 & $23-45$ & - & - & - & $23-45$ & 40 \\
\hline 2014 & - & - & - & - & - & - & - & - \\
\hline 2015 & - & - & - & - & - & - & - & - \\
\hline
\end{tabular}

Contamination of Aflatoxin B1, B2, G1, G2 and total AFs range in Peanut cake

Table 18 Parboiled rice

\begin{tabular}{|c|c|c|c|c|c|c|c|c|}
\hline Duration & $\begin{array}{c}\text { no of } \\
\text { analyzed } \\
\text { samples }\end{array}$ & $\begin{array}{c}\text { no of } \\
\text { positive } \\
\text { samples }\end{array}$ & $\begin{array}{c}\text { Range } \\
(\mathrm{B} 1) \\
(\mathrm{ppb})\end{array}$ & $\begin{array}{c}\text { Range } \\
(\mathrm{B} 2) \\
(\mathrm{ppb})\end{array}$ & $\begin{array}{c}\text { Range } \\
(\mathrm{G} 1) \\
(\mathrm{ppb})\end{array}$ & $\begin{array}{c}\text { Range } \\
(\mathrm{G} 2) \\
(\mathrm{ppb})\end{array}$ & $\begin{array}{c}\text { Range } \\
\text { (total) } \\
(\mathrm{ppb})\end{array}$ & $\begin{array}{c}\text { Percent of } \\
\text { positive } \\
\text { samples (\%) }\end{array}$ \\
\hline 2008 & - & - & - & - & - & - & - & - \\
\hline 2009 & - & - & - & - & - & - & - & - \\
\hline 2010 & - & - & - & - & - & - & - & - \\
\hline 2011 & - & - & - & - & - & - & - & - \\
\hline 2012 & 15 & 8 & $0.3-3$ & - & - & - & $0.3-3$ & 53 \\
\hline 2013 & 10 & 2 & $2-5$ & - & - & - & $2-5$ & 20 \\
\hline 2014 & 53 & 38 & $0.8-9$ & $0.4-7$ & - & - & $0.8-12$ & 72 \\
\hline 2015 & 53 & 36 & $2-9$ & $2-2$ & - & - & $2-14$ & 68 \\
\hline
\end{tabular}

Contamination of Aflatoxin B1, B2, G1, G2 and total AFs range in Parboiled rice

Table 19 summarize the aflatoxin contamination of Myanmar agricultural commodities

\begin{tabular}{|c|c|c|c|}
\hline Types of sample & no. of analyzed samples & no.of positive samples & Percent of positive samples (\%) \\
\hline White rice & 514 & 344 & 67 \\
\hline Broken rice & 242 & 169 & 70 \\
\hline Green mung bean & 334 & 84 & 25 \\
\hline Black sesame seed & 955 & 203 & 21 \\
\hline White sesame & 110 & 35 & 32 \\
\hline Toor whole & 256 & 56 & 22 \\
\hline Maize & 137 & 97 & 71 \\
\hline Butter bean & 515 & 164 & 32 \\
\hline Black matpe & 666 & 104 & 16 \\
\hline Peyin & 58 & 23 & 40 \\
\hline Red kidney bean & 20 & 9 & 45 \\
\hline Sultanipya & 29 & 10 & 3 \\
\hline Black eyed bean & 20 & 5 & 25 \\
\hline Bocate bean & 25 & 8 & 32 \\
\hline Soya bean & 9 & 3 & 33 \\
\hline Chicken pea bean & 7 & 4 & 57 \\
\hline Peanut cake & 14 & 7 & 50 \\
\hline Parboiled rice & 131 & 84 & 64 \\
\hline TOTAL & 4042 & 1409 & 35 \\
\hline
\end{tabular}

Aflatoxin contamination of Myanmar agricultural commodities in 2008 -2015 
AFB1 contaminated level of some cereal grains and oilseed samples such as white rice, broken rice, parboiled rice, maize, black sesame seed and white sesame seed were higher than the regulated level of Codex Alimentarius Commission and European Union (EU). This is possible due to the following factors.

Along the supply chain, these commodities are affected by various actors who have variable capacities to handle mass production, manage in transportation, and also quality control. Due to the lack of the knowledge related to quality standards among the actors in the supply chain in Myanmar, quality assessment is based on personal experiences, and visual and sensory inspections of the seeds. Moreover, there is no laboratory available to quickly perform basic tests, such as moisture content, near the commodity producing regions.

The farmers knowledge is very important for exporters because the quality of the end products depend on the quality of the starting products. Some farmers lack of modernized technologies and knowledge about input quality and therefore their products need proper wide-ranging services. As such, the farmer's socioeconomic position needs to be promoted for a smooth and fair export process. One of the other important considerations for farmers is to consider alternatives for some of traditional theories concerning post - harvest technologies and to follow the good agricultural practices (GAP).

Improper handling practices during the storage, re-wetting during transportation, insect infection during long-term storage, absorption of moisture during the rainy season and precipitation events are also factors that influence aflatoxin contamination in agricultural commodities in Myanmar.

\section{Conclusion}

The analysis of aflatoxin contamination in Myanmar agricultural commodities during the eight-year study gives the percentage of contaminated samples and their contaminated levels year by year of each item. The samples were collected from various parts of the country during different weather conditions. The significant differences in AFB1 levels are not only based on climate variation but also the result of differences in harvesting, storage, transportation and handling conditions. The data from past eight-year study did not show correlation to each other. However, aflatoxin contamination levels for more recent crop year were lower than those found for previous crop years. In the future, proper monitoring programs and surveys should be conducted to ensure food safety for consumers and to meet the requirements of international markets.

\section{Acknowledgements}

The author would like to thank Director General, Director and Deputy Directors of Consumer Affairs Department (Ministry of Commerce), for encouraging attendance to the ISMYCO 2016. The author would also like to thank Dr. Masahiro Nakajima: Manager, Food Department of the Nagoya City Public Health Research Institute for his invitation to attend ISMYCO 2016 and all the colleagues of CTQM (HLEGU), especially Daw Yin Yin Swe for providing previous technical data.

\section{References}

1) Data Source: Mycotoxin Laboratory from CTQM (HLEGU)

2) Romer, T.R., J. Assoc. Off. Anal. Chem., 58, 500-506 (1975) 